\title{
Resultados de um projeto no ensino interprofissional: capacitação sobre acolhimento para Agentes Comunitários de Saúde
}

\author{
Outcomes of a project in interprofissional education: training on user embracement for \\ Community Health Agents
}
Resultados de un proyecto en la enseñanza interprofesional: capacitación sobre acogida para Agentes Comunitarios de Salud

Najara Barbosa da Rocha ${ }^{1()}$, Amanda Meira Saraiva ${ }^{2}$, Sabrina Ferreira Cruz $^{3}$, Ana Tereza Franchin ${ }^{4}$, Luiz Fernando Lolli ${ }^{5}$, Mitsue Fujimaki ${ }^{6}$

\footnotetext{
${ }^{1}$ Doutora em Odontologia Preventiva e Social pela Faculdade de Odontologia de Araçatuba; Docente no Departamento de Odontologia - Universidade Estadual de Maringá - PR

${ }^{2}$ Residente em Odontologia Saúde Coletiva e da Família; Universidade Estadual de Maringá - PR

${ }^{3}$ Residente em Odontologia Saúde Coletiva e da Família; Universidade Estadual de Maringá- PR

${ }^{4}$ Médica da Família do Município de Sarandi- PR

${ }^{5}$ Doutor em Odontologia Preventiva e Social pela Universidade Estadual Paulista Júlio de Mesquita Filho; Docente no Departamento de Odontologia - Universidade Estadual de Maringá- PR

${ }^{6}$ Doutora em Odontologia pela Universidade Estadual de Campinas; Docente no Departamento de Odontologia - Universidade Estadual de Maringá- PR
}

\section{RESUMO}

A classificação de risco do paciente no setor de acolhimento é uma ferramenta que organiza a fila de espera para o melhor atendimento do paciente. Assim, objetivou-se avaliar os resultados de um projeto aplicado na disciplina interprofissional sobre capacitação em relação ao acolhimento com classificação de risco (ACR) das agentes comunitárias de saúde de uma Unidade Básica de Saúde de um município do norte do Paraná. É uma pesquisa descritiva, exploratória, prospectiva, com abordagem quantitativa. Aplicou-se um questionário com dados demográficos e sobre o ACR. Os resultados demonstraram que antes da capacitação as agentes não prestavam suporte ao usuário de forma segura e confortável, atendiam por ordem de chegada e sem conhecimento suficiente. Após, houve melhora no entendimento 
e realização do acolhimento. A educação permanente é ideal para a melhoria do atendimento na Atenção Primária, e deve fazer parte da rotina dos profissionais de saúde para qualificação da organização da atenção.

Palavras-chave: Acolhimento, Sistema Único de Saúde, Atenção Primária à Saúde.

\begin{abstract}
The risk classification of the patient in the embracement department is a tool that organizes the waiting line for best patient care. The aim of this study was to evaluate the results of a project applied in an interprofessional discipline on the qualification regarding the user embracement with risk classification (UERC) of the community health agents of a basic health unit of a municipality in the north of the state of Paraná. This is a descriptive, exploratory, prospective study with a quantitative approach. A questionnaire with demographic data and another about UERC was applied. Before the training the agents did not provide safe and comfortable support for the user, attending them in the order of arrival and without sufficient knowledge. After the training there was improvement on the understanding and achievement of the user embracement. Permanent education in health is optimal for Primary Care improvement of attendance, and should be part of the routine of health professionals to qualify care organization.
\end{abstract}

Keywords: User Embracement, Unified Health System, Primary Health Care.

\title{
RESUMEN
}

La clasificación de riesgo del paciente en el sector de acogimiento es una herramienta que organiza la fila de espera para mejor atención al paciente. El objetivo fue evaluar los resultados del proyecto en la asignatura interprofesional sobre capacitación en relación al acogimiento con clasificación de riesgo (ACR) de los agentes comunitarios de salud de una Unidad Básica de Salud de una ciudad del norte de Paraná. Es una investigación descriptiva, exploratoria, prospectiva, con abordaje cuantitativo. Se aplicó una encuesta con datos demográficos y sobre el ACR. Los resultados comprobaron que antes de la capacitación los agentes no prestaban soporte al usuario de forma segura y confortable, atendían por orden de llegada y sin conocimiento suficiente. Después, hubo una mejora en el entendimiento y realización de la acogida. La educación permanente es ideal para mejorar la atención en la Atención Primaria, debe formar parte de la rutina de los profesionales de salud para calificación de la organización de la atención.

Palabras clave: Acogimiento, Sistema Único de Salud; Atención Primaria de Salud.

\section{INTRODUÇÃo}

A Estratégia Saúde da Família (ESF) se baseia em uma equipe multiprofissional da Atenção Primária (AP), que possui, no mínimo, médico, enfermeiro, auxiliar ou técnico de enfermagem e agentes comunitários de saúde $(\mathrm{ACS})^{1}$. Os agentes comunitários de saúde são de fundamental importância dentro da Atenção Primária, pois são eles os responsáveis pelo primeiro contato com os usuários do serviço público de saúde em seus domicílios, sendo capazes de fortalecer o vínculo entre o serviço e a comunidade ${ }^{2}$. 
Mais de $85 \%$ dos problemas de saúde da população podem e devem ser resolvidos na Atenção Primária, ou seja, em sua porta de entrada, na Unidade Básica de Saúde (UBS). Entretanto não é isso que se nota no cotidiano, mas uma grande espera e superlotação para o atendimento nos serviços públicos de saúde ${ }^{3}$. Alguns problemas poderiam ser resolvidos ou amenizados pela prática do acolhimento, a partir da avaliação e classificação de risco dos pacientes ${ }^{4,5}$.

Segundo a Política Nacional de Humanização (Humaniza SUS $^{6}$, o acolhimento expressa uma ação de aproximação, ou seja, uma atitude de inclusão que deve estar apoiada na avaliação e classificação de risco, ferramenta que organiza a fila de espera e propõe outra ordem de atendimento que não a ordem de chegada ${ }^{6}$, visando eficiência e efetividade organizacional ${ }^{7,8}$. A partir disso, os pacientes que chegam ao serviço de saúde são atendidos mediante critérios clínicos, sendo priorizado o cuidado em relação à gravidade, potencial de risco e/ou; grau de sofrimento das pessoas, por um processo dinâmico em saúde, garantindo maior qualidade no atendimento dos serviços de emergência9.

Deve-se ressaltar que o acolhimento não está restrito a um setor, a uma hora e a um profissional, sendo caracterizado por postura ética desde o momento em que o usuário chega à unidade de saúde e faz o primeiro contato com aquele que imagina ser o atendente que o ouvirá e o acolherá ${ }^{10}$.

Tendo em vista que o ACS é membro importante dentro do acolhimento e também na implementação do Sistema Único de Saúde (SUS), fortalecendo a integração entre os serviços de saúde da $\mathrm{AP}$ e a comunidade, o Ministério da Saúde estabelece a necessidade de capacitação permanente dos agentes ${ }^{2}$. Entretanto, atualmente observa-se a ocorrência de falhas na execução das diretrizes propostas pela Política Nacional de Humanização em diversas UBS do Brasil, visto que o acolhimento por muitas vezes não é implantado e os profissionais envolvidos não possuem capacitação específica, demonstrando, assim, uma falha na adequação ao novo modelo assistencial enfatizado pela doutrina do SUS ${ }^{1,11,12,13}$.

Sendo assim, o objetivo deste trabalho foi avaliar o conhecimento sobre o acolhimento com classificação de risco dos ACS em uma UBS de um município do Paraná, antes e após a capacitação sobre o referido assunto.

\section{MÉTODO}

Realizou-se uma pesquisa descritiva, exploratória, prospectiva, com abordagem quantitativa com agentes comunitários de saúde em uma UBS de um município paranaense, que é considerada referência, pois recebe a maior parte dos atendimentos de saúde desta cidade.

Um estudo piloto foi realizado, para aferição das dificuldades encontradas, capacitação e calibração dos pesquisadores e do questionário aplicado. A coleta de dados foi realizada por um único entrevistador calibrado, sem prejuízos às atividades dos funcionários. Os objetivos e a metodologia da pesquisa foram explicados às ACS, e aquelas que aceitaram participar do estudo assinaram um termo de consentimento livre e esclarecido. Mesmo em caso de recusa à participação no estudo, as agentes foram esclarecidas que poderiam participar da capacitação sobre o acolhimento com avaliação e classificação de risco.

O instrumento de coleta de dados ${ }^{14,15}$ foi composto por um questionário contendo dados sociodemográficos e outro pré-testado ${ }^{14}$ composto por questionamentos relacionados ao AACR, ambos aplicados antes do início das atividades e uma semana após o término da capacitação. Todas as ACS da unidade foram convidadas a participar do estudo e nenhuma se recusou.

Esta capacitação foi resultante da aplicação de um projeto de intervenção, que fez parte da avaliação final de uma disciplina interprofissional da área 
da saúde de alunos da Universidade Estadual de Maringá. Os alunos desenvolveram a capacitação, orientadas pelo tutor (professor) e preceptor da turma, pautada no referencial teórico disponibilizado pelo Ministério da Saúde ${ }^{3,16}$ e de acordo com a Política Nacional de Humanização ${ }^{6}$, e foi centrada na metodologia de ensino aprendizagem ativa por meio de rodas de conversas e dramatização da realidade encontrada na UBS. A principal diretriz da capacitação foi fazer com que todas as agentes reconhecessem e ficassem conscientes do seu papel dentro da unidade, juntamente com a prática do acolhimento, que é uma ferramenta indispensável para o bom atendimento, e que ambas as partes, tanto o atendente como o atendido, pudessem ser beneficiadas com tal prática. Foi enfatizada a importância de uma equipe multiprofissional com postura acolhedora e com objetivo comum: o bemestar físico, emocional e psicológico da população. É relevante também ressaltar a necessidade de conscientização da população em relação ao processo de tomada de decisão no AACR, para que se torne possível melhorar o funcionamento da UBS de forma significativa e sem prejuízos à comunidade ${ }^{16}$. A capacitação ocorreu a partir de três contatos realizados ao longo de três semanas. A primeira atividade consistiu em um encontro com as ACS, em forma de "roda de conversa" associada a um "café", no local destinado às refeições dos funcionários.

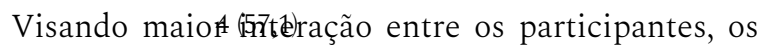
mesmos se apresentaram e em seguida, objetivando o desenvolvimento de uma discussão, foram indagados em relação à importância do trabalho desenvolvido por eles para a $\mathrm{a}^{(5 \mathrm{~S} B S}$ e como pensavam que deveriam agir dentro da ESF. A partir destas respostas, foi possível explicitar as suas atribuições e funções, buscando evidenciar a relevância do trabalho como fundamento e base do funcionamento da AP. No segundo encontro, o tema em pauta foi acolhimento e buscou-se a construção de uma discussão acerca desta prática, 6 (8ligando as opiniões das agentes a respeito do acolhimento com as definições, diretrizes e recomendações apresentadas pela literatura - principalmente a Política Nacional de Humanização do SUS - enaltecendo sua importância e suas contribuições para a melhoria do serviço de saúde. No encontro final, foi utilizada a metodologia problematizadora, tendo como referência o Método do Arco de Charlez Maguerez por possibilitar a participação ativa dos participantes, colocandoos, não como meros receptores, mas como fonte de conhecimentos e experiências, engajando-os na identificação e solução de problemas dos seus $\operatorname{cotidianos}^{17}$. Utilizou-se o método de dramatização das atividades no acolhimento e das ACS no atendimento a domicílio ${ }^{18}$. O objetivo geral do teatro foi conscientizar as funcionárias a respeito do seu papel e a importância da ACS no trabalho comunitário em saúde, estimulando-as a atuar, de forma ética e proativa, na promoção da saúde, na comunicação interpessoal e no desenvolvimento do trabalho em equipe, em prol da melhoria da atenção de saúde à população local.

Após a coleta dos dados, os formulários foram digitados e processados no programa Epi Info $^{19}$. Após a digitação, os dados foram novamente conferidos, a fim de corrigir todos os erros inerentes a este processo por outro pesquisador. Os dados quantitativos foram analisados estatisticamente no Programa Bioestat ${ }^{20}$. A análise estatística incluiu a análise descritiva dos resultados apresentados em tabelas.

O projeto de pesquisa foi encaminhado para o Comitê de Ética em Pesquisa em Seres Humanos da Universidade Estadual de Maringá, por meio da plataforma Brasil e aprovado sob o número de parecer 1.666.395.

\section{RESULTADOS}

A coleta de dados antes e depois da capacitação foi feita com 7 agentes da UBS, cuja idade média era $32,1( \pm 7.3)$ anos, sendo que todas eram casadas e do sexo feminino e quatro agentes apresentavam mais de 8 anos de estudo (57,1\%). 
Tabela 1 - Distribuição numérica e percentual das ACS de uma UBS do Norte do Paraná, de acordo com suas características sociodemográficas.

\begin{tabular}{lcc}
$\begin{array}{l}\text { Características } \\
\text { Idade }\end{array}$ & N & \% \\
Até 30 anos & 4 & 57,1 \\
\hline $\begin{array}{l}\text { 31 anos ou mais } \\
\text { Situação conjugal } \\
\text { Casado }\end{array}$ & 3 & 43,9 \\
\hline $\begin{array}{l}\text { Solteiro } \\
\text { Escolaridade }\end{array}$ & 7 & 100 \\
$\quad$ Até 8 anos de estudo & 0 & 0 \\
\hline $\begin{array}{l}\text { Mais de } 8 \text { anos } \\
\text { Sexo }\end{array}$ & 3 & 43,9 \\
$\quad$ Feminino & 4 & 57,1 \\
\hline Masculino & 7 & 100 \\
\hline
\end{tabular}

Fonte: Saraiva et al. (2018)
Todas as agentes foram capacitadas e antes da capacitação responderam o questionário sobre $\mathrm{o}$ acolhimento. As respostas antes e após a capacitação se encontram na tabela 2.

Antes da capacitação, os resultados indicaram que um pouco mais da metade das agentes concordaram com as vantagens do acolhimento em deixar um ambiente aconchegante $(57,1 \%)$ e promover conforto ao usuário e acompanhante (57,1\%).

Em relação ao espaço físico da UBS, a maioria concordou que para consulta médica e de enfermagem, o espaço não proporcionava privacidade ao usuário $(85,7 \%)$, não era suficiente para acomodar o acompanhante $(85,7 \%)$ e a sinalização no ambiente para direcionar o atendimento na UBS não estava clara (57,1\%).

Tabela 2 - Distribuição numérica e percentual das respostas das ACS de uma UBS de um município do Paraná antes e após a capacitação sobre AACR.

\begin{tabular}{|c|c|c|c|c|c|c|c|}
\hline \multirow[t]{2}{*}{ Questões } & \multirow{2}{*}{$\begin{array}{l}\text { Houve melhoria } \\
\text { do conhecimento } \\
\text { das agentes } \\
\text { antes e depois da } \\
\text { capacitação? }\end{array}$} & \multicolumn{3}{|c|}{$\begin{array}{c}\text { Antes da capacitação } \\
\mathbf{n}(\%)\end{array}$} & \multicolumn{3}{|c|}{$\begin{array}{c}\text { Depois da capacitação } \\
\mathbf{n}(\%)\end{array}$} \\
\hline & & Concordo & Neutro & Discordo & Concordo & Neutro & Discordo \\
\hline $\begin{array}{l}\text { 1) A estrutura física para } \\
\text { o Acolhimento com } \\
\text { Classificação de Risco } \\
\text { promove conforto ao } \\
\text { usuário e acompanhante }\end{array}$ & Sim & $4(57,1)$ & $1(14,3)$ & $2(28,6)$ & $4(57,1)$ & $1(14,3)$ & $2(28,6)$ \\
\hline $\begin{array}{l}\text { 2) O Acolhimento com } \\
\text { Classificação de Risco torna } \\
\text { o ambiente mais acolhedor } \\
\text { e humano }\end{array}$ & Sim & $4(57,1)$ & $1(14,3)$ & $2(28,6)$ & $4(57,1)$ & $1(14,3)$ & $2(28,6)$ \\
\hline $\begin{array}{l}\text { 3) Não ocorrem reuniões } \\
\text { e treinamentos periódicos } \\
\text { para os trabalhadores que } \\
\text { atuam no Acolhimento com } \\
\text { Classificação de Risco }\end{array}$ & Sim & $6(85,7)$ & $0(0)$ & $1(14,3)$ & $6(85,7)$ & $0(0)$ & $1(14,3)$ \\
\hline $\begin{array}{l}\text { 4) O espaço físico } \\
\text { para consulta médica } \\
\text { e de enfermagem não } \\
\text { proporcionam privacidade } \\
\text { ao usuário }\end{array}$ & Sim & $6(85,7)$ & $1(14,3)$ & $0(0)$ & $6(85,7)$ & $1(14,3)$ & $0(0)$ \\
\hline
\end{tabular}




\begin{tabular}{|c|c|c|c|c|c|c|c|}
\hline $\begin{array}{l}\text { 5) O espaço físico não é } \\
\text { suficiente para acolher o } \\
\text { acompanhante }\end{array}$ & Sim & $6(85,7)$ & $1(14,3)$ & $0(0)$ & $6(85,7)$ & $1(14,3)$ & $0(0)$ \\
\hline $\begin{array}{l}\text { 6) A sinalização do ambiente } \\
\text { é clara e suficiente para } \\
\text { o direcionamento do } \\
\text { usuário ao atendimento } \\
\text { no Acolhimento com } \\
\text { Classificação de Risco }\end{array}$ & Sim & $2(28,6)$ & $1(14,3)$ & $4(57,1)$ & $2(28,6)$ & $1(14,3)$ & $4(57,1)$ \\
\hline $\begin{array}{l}\text { 7) Os trabalhadores que } \\
\text { atuam no Acolhimento com } \\
\text { Classificação de Risco não se } \\
\text { comunicam frequentemente } \\
\text { com toda a equipe do setor }\end{array}$ & Sim & $3(43,9)$ & $0(0)$ & $4(57,1)$ & $3(43,9)$ & $0(0)$ & $4(57,1)$ \\
\hline $\begin{array}{l}\text { 8) Todos os pacientes não } \\
\text { graves que procuram por } \\
\text { atendimento nesse serviço } \\
\text { de emergência passam } \\
\text { pelo Acolhimento com } \\
\text { Classificação de Risco }\end{array}$ & Sim & $3(43,9)$ & $1(14,3)$ & $3(43,9)$ & $3(43,9)$ & $1(14,3)$ & $3(43,9)$ \\
\hline $\begin{array}{l}\text { 9) As condutas a serem } \\
\text { tomadas descritas no } \\
\text { protocolo de Acolhimento } \\
\text { com Classificação de Risco } \\
\text { não são conhecidas por todos } \\
\text { que trabalham no setor }\end{array}$ & Sim & $6(85,7)$ & $0(0)$ & $1(14,3)$ & $6(85,7)$ & $0(0)$ & $1(14,3)$ \\
\hline $\begin{array}{l}\text { 10) As lideranças desse } \\
\text { serviço discutem com os } \\
\text { funcionários de forma } \\
\text { democrática e acolhedora as } \\
\text { propostas de mudanças no } \\
\text { serviço de Acolhimento com } \\
\text { Classificação de Risco }\end{array}$ & Sim & $1(14,3)$ & $0(0)$ & $6(85,7)$ & $1(14,3)$ & $0(0)$ & $6(85,7)$ \\
\hline $\begin{array}{l}\text { 11) Os Profissionais que } \\
\text { atuam no Acolhimento } \\
\text { com Classificação de Risco } \\
\text { contribuem para que o } \\
\text { usuário se sinta seguro e } \\
\text { confortável }\end{array}$ & Sim & $2(28,6)$ & $1(14,3)$ & $4(57,1)$ & $2(28,6)$ & $1(14,3)$ & $4(57,1)$ \\
\hline $\begin{array}{l}\text { 12) O Fluxograma de } \\
\text { atendimento do Acolhimento } \\
\text { com Classificação de Risco } \\
\text { é discutido com a equipe } \\
\text { e avaliado periodicamente } \\
\text { quanto à clareza e } \\
\text { objetividade }\end{array}$ & Sim & $1(14,3)$ & $2(28,6)$ & $4(57,1)$ & $1(14,3)$ & $2(28,6)$ & $4(57,1)$ \\
\hline $\begin{array}{l}\text { 13) O atendimento primário } \\
\text { ao usuário ocorre de acordo } \\
\text { com a gravidade do caso e } \\
\text { não de acordo com a ordem } \\
\text { de chegada }\end{array}$ & Sim & $2(28,6)$ & $2(28,6)$ & $3(43,9)$ & $2(28,6)$ & $2(28,6)$ & $3(43,9)$ \\
\hline
\end{tabular}




\begin{tabular}{|c|c|c|c|c|c|c|c|}
\hline $\begin{array}{l}\text { 14) O usuário que não } \\
\text { corre risco imediato, assim } \\
\text { como a seus familiares, é } \\
\text { informado sobre o tempo } \\
\text { provável de espera pelo } \\
\text { atendimento }\end{array}$ & Sim & $3(43,9)$ & $1(14,3)$ & $3(43,9)$ & $3(43,9)$ & $1(14,3)$ & $3(43,9)$ \\
\hline $\begin{array}{l}\text { 15) Os profissionais que } \\
\text { atuam no Acolhimento } \\
\text { com Classificação de } \\
\text { Risco estão treinados } \\
\text { para atender ao usuário e } \\
\text { acompanhante de forma } \\
\text { acolhedora e humana }\end{array}$ & Sim & $2(28,6)$ & $3(43,9)$ & $2(28,6)$ & $2(28,6)$ & $3(43,9)$ & $2(28,6)$ \\
\hline $\begin{array}{l}\text { 16) Nesse serviço, } \\
\text { a humanização no } \\
\text { atendimento não se faz } \\
\text { presente em todas as } \\
\text { etapas do atendimento ao } \\
\text { usuário }\end{array}$ & Sim & $4(57,1)$ & $2(28,6)$ & $1(14,3)$ & $4(57,1)$ & $2(28,6)$ & $1(14,3)$ \\
\hline $\begin{array}{l}\text { 17) A equipe } \\
\text { multiprofissional que atua } \\
\text { nesse serviço trabalha de } \\
\text { forma integrada e atenta às } \\
\text { necessidades dos usuários }\end{array}$ & Sim & $3(43,9)$ & $1(14,3)$ & $3(43,9)$ & $3(43,9)$ & $1(14,3)$ & $3(43,9)$ \\
\hline $\begin{array}{l}\text { 18) Os usuários que } \\
\text { passam pelo Acolhimento } \\
\text { com Classificação de } \\
\text { Risco e aguardam pelo } \\
\text { atendimento médico } \\
\text { não são reavaliados } \\
\text { periodicamente }\end{array}$ & Sim & $2(28,6)$ & $3(43,9)$ & $2(28,6)$ & $2(28,6)$ & $3(43,9)$ & $2(28,6)$ \\
\hline $\begin{array}{l}\text { 19) Mesmo com o } \\
\text { Acolhimento com } \\
\text { Classificação de Risco } \\
\text { os pacientes graves não } \\
\text { são priorizados para o } \\
\text { atendimento }\end{array}$ & Sim & $4(57,1)$ & $1(14,3)$ & $2(28,6)$ & $4(57,1)$ & $1(14,3)$ & $2(28,6)$ \\
\hline $\begin{array}{l}\text { 20) Os casos de baixa } \\
\text { complexidade não são } \\
\text { encaminhados à rede } \\
\text { básica de saúde * }\end{array}$ & Sim & $2(28,6)$ & $1(14,3)$ & $4(57,1)$ & $2(28,6)$ & $1(14,3)$ & $4(57,1)$ \\
\hline $\begin{array}{l}\text { 21) Os profissionais } \\
\text { que atuam nesse setor } \\
\text { se sentem satisfeitos } \\
\text { com a implantação } \\
\text { do Acolhimento com } \\
\text { Classificação de Risco no } \\
\text { atendimento }\end{array}$ & Sim & $2(28,6)$ & $0(0)$ & $5(71,4)$ & $2(28,6)$ & $0(0)$ & $5(71,4)$ \\
\hline
\end{tabular}

Fonte: Saraiva et al. (2018)

* Um participante não respondeu a esta questão no segundo questionário. 
A maioria consentiu que não havia treinamento para a equipe sobre acolhimento (85,7\%), não conheciam as condutas a serem tomadas para acolher o usuário $(85,7 \%)$ e as chefias não discutiam com os funcionários mudanças para melhoria da AACR (85,7\%).

Poucas funcionárias admitiram que atendiam de forma humanizada o paciente $(28,6 \%)$ e que o usuário de baixo risco era informado sobre o tempo provável de espera pelo atendimento (43,9\%).

A maioria das servidoras estava insatisfeita com a implantação do acolhimento (71,4\%) e 57,1 \% afirmaram que a humanização não estava presente no atendimento em todas as etapas do atendimento ao usuário na UBS.

As agentes discordaram $(43,9 \%)$ que a UBS utilizava a priorização de pacientes de acordo com a necessidade e não com a ordem de chegada, e concordaram que a UBS não privilegiava os pacientes graves quando chegavam para o atendimento $(57,1 \%)$.

Após a capacitação (tabela 2), foi verificada uma melhoria significativa em todos os quesitos em relação à AACR. Todas concordaram que a estrutura para o acolhimento na UBS promove conforto ao usuário e acompanhante, $85,7 \%$ acharam que proporciona privacidade ao usuário e concordaram que o espaço era suficiente para acolher o acompanhante $(85,7 \%)$, e $71,4 \%$ concordaram que a sinalização na UBS para orientar o paciente é suficiente.

Houve uma grande melhora $(57,1 \%)$ nas respostas sobre a ocorrência de reuniões e treinamentos sobre acolhimento na UBS, a comunicação melhorou significativamente $(42,9 \%)$ entre liderança e funcionários, bem como ocorreu melhora $(42,9 \%)$ da discussão sobre o fluxograma de atendimento.

Após a capacitação o número de profissionais que atuavam na AACR e que se sentiram aptas a atender ao usuário e acompanhante de forma acolhedora e humana aumentou $42,9 \%$, e apenas uma ACS $(14,3 \%)$ se mostrou indiferente com a implantação do acolhimento. Também foi possível notar melhoria
$(42,9 \%)$ em relação à humanização em todas as etapas do atendimento ao usuário.

A maioria $(71,4 \%)$ concordou que os pacientes não graves passam por acolhimento com classificação de risco e o atendimento é feito de acordo com a gravidade dos casos $(85,7 \%)$.

\section{DISCUSSÃo}

A consolidação da prática do acolhimento é um desafio, pois não depende do desejo apenas do trabalhador, gestor e/ou usuário, pois o produto da ação do trabalhador dependerá do rearranjo dos diferentes elementos estruturais, de recursos humanos e também do processo assistencial estabelecido nas instituições de saúde ${ }^{21}$.

O acolhimento é compreendido como processo, resultado de práticas de saúde, produto da relação entre trabalhadores de saúde e usuários, constituindose, assim, em um conjunto de atos executados de modos distintos no momento do atendimento, envolvendo posturas e concepções adotadas pelo trabalhador na identificação das demandas e necessidades dos usuários ${ }^{4}$. $\mathrm{O}$ acolhimento é a porta de entrada do serviço de saúde para tornar-se mais humano.

A população do estudo foi composta por mulheres, casadas, jovens e com nível médio de escolaridade. Estes resultados corroboram com Versa et al. ${ }^{15}$.

Neste estudo foi verificado que a sinalização antes da capacitação era insuficiente para orientar o paciente de forma clara dentro da UBS e houve uma melhora na segunda entrevista. A preocupação para com o usuário pode ser observada também pela forma com que se utiliza da sinalização e se informa a localização das salas e setores de atendimento, isto é, como se lança mão da tecnologia dura: placas, cartazes, painéis informativos e outros. Desde a entrada, é preciso destacar placas e ter pessoas nos setores que informem sobre o acolhimento corretamente ${ }^{15}$. 
Matumoto et al. ${ }^{4}$ diz que o serviço como um todo, por meio de seus trabalhadores, deve considerar a sinalização tal como se encontra, orientando os usuários, segundo suas necessidades; ficando, assim, atentos para identificar, dentre o conjunto de usuários, aqueles que apresentam dificuldades em se localizar espacialmente na unidade de saúde, seja por limitação de escolaridade, seja por dificuldades circunstanciais como dor, ansiedade, medo, em um exercício constante da equidade e acessibilidade.

Considera-se que a estrutura física da UBS deve proporcionar condições adequadas ao atendimento dos pacientes e seus acompanhantes, pois este é um dos requisitos essenciais propostos pela Política Nacional de Humanização ${ }^{6}$, no entanto no primeiro momento as ACS consideravam que o espaço físico era inadequado para a prática do acolhimento. Após a capacitação, foi possível notar melhorias neste quesito, pois além da transmissão de conhecimento, houve uma mudança de estrutura e localidade desta UBS, sendo que esta foi transferida para um lugar mais amplo, sinalizado e mais humanizado segundo as ACS.

Historicamente, o atendimento à saúde em instituições públicas tem sido determinado pela ordem de chegada do usuário, sem avaliação prévia dos $\operatorname{casos}^{22,23}$. Isso, sem dúvida, é preocupante, pois resulta em demora do atendimento e faz com que os pacientes sejam expostos, desnecessariamente, a riscos e/ou complicações, perdendo-se a possibilidade de intervenção precoce e manejo adequado da sua condição patológica ${ }^{6,24}$. No sentido de minimizar essa distorção e ineficácia do sistema, a implantação de um sistema de acolhimento torna-se urgente, como forma de garantir uma assistência de qualidade e segurança para os usuários dos serviços.

$\mathrm{O}$ atendimento por ordem de chegada aumenta o tamanho das filas, causa demora no atendimento, o paciente fica insatisfeito e ainda pode ter problemas com o risco da espera do atendimento ${ }^{4,15}$. Era este o cenário encontrado na UBS antes da capacitação, e após, esta conduta deixou de ser adotada. Foi possível verificar que a capacitação realizada teve influência positiva sobre o processo e classificação de risco dos pacientes, o usuário em caso grave passou a ser priorizado para o atendimento, enquanto o que não corre risco imediato, assim como seus familiares, agora é informado sobre o tempo provável de espera pelo atendimento.

O profissional de saúde dentro da Unidade Básica tem que ser responsável pelo encaminhamento aos serviços de maior densidade tecnológica. O acolhimento não é apenas como recepção do usuário em um serviço de saúde qualquer, mas como ação de responsabilização do trabalhador pelo usuário durante toda sua permanência no serviço de saúde ${ }^{25}$. Foi verificado que antes o paciente não era encaminhado e após a capacitação houve uma melhora nesse processo.

$\mathrm{O}$ acolhimento considerado como um processo a ser desenvolvido antes, durante e após o atendimento deve ser realizado por todos os profissionais, sendo que cada um deles contribuirá positivamente para o desenvolvimento dessa ferramenta assistencial e humanizadora dentro de sua área de atuação ${ }^{26}$. Ou seja, é como instrumento de eficácia no atendimento que envolve todos os membros da equipe multiprofissional e não admite negligência ${ }^{10}$.

Atualmente observa-se que existe uma grande lacuna pelo que foi proposto pelo Ministério da Saúde e pelo que é executado nas unidades de saúde, pois os profissionais envolvidos não passam por uma capacitação específica ${ }^{11}$. Os profissionais do setor do acolhimento devem estar preparados e capacitados para atender de forma humanizada os pacientes. Fazse necessária uma educação permanente aprimorando e fornecendo dados para formar profissionais aptos com perfil para atender a população, de forma multiprofissional, com resolubilidade e respeito mútuo entre os profissionais e o paciente.

Trindade ${ }^{10}$ cita que para o acolhimento ser efetivo basta que a equipe seja treinada, capacitada, valorizada, sejam oferecidos salários dignos, divisão 
de trabalho, organização, vínculo, comprometimento com a comunidade dentro da ética e, acima de tudo, respeito.

No geral, houve melhora na avaliação da qualidade no acolhimento desta UBS em todos os quesitos avaliados, evidenciando que a educação permanente é importante e se faz necessária para o processo de trabalho da equipe.

Os resultados deste estudo foram significativos, porém devem-se ressaltar algumas limitações. A primeira limitação foi sobre o desenho do estudo ser do tipo transversal e por isso ter alguns vieses como de memória ou desejabilidade social e não conseguir fornecer maior evidência nos resultados, havendo a necessidade de realização de estudos longitudinais. Outra limitação seria o tipo de coleta de dados que foi por questionário auto-aplicado. Novas pesquisas longitudinais deverão ser conduzidas para melhorar as evidências deste achado. Ainda é possível citar a mudança na localização da UBS entre as aplicações do primeiro e segundo questionários, fator que pode gerar conflitos em alguns quesitos. Contudo, considera-se que os resultados deste estudo possam contribuir para novos processos de capacitação sobre acolhimento, no sentido de promover melhorias na atuação da equipe e consequentemente na qualidade do atendimento ao usuário, diminuindo filas.

\section{CONCLUSÕES}

Os resultados sugerem que antes da capacitação os funcionários mostraram disposição em atender o paciente de forma humanizada, porém não havia conhecimento, ou este era insuficiente, sobre acolhimento com classificação de risco. Verificaram-se falhas no apoio e treinamento por parte da coordenação da UBS, bem como falta de estrutura física para o funcionamento adequado do acolhimento.

A capacitação desencadeou melhorias significativas, seja no AACR, na estrutura e até mesmo no relacionamento entre lideranças e funcionários. A educação permanente em saúde é ideal para a melhoria do atendimento na atenção primária, deve ser estimulada e fazer parte da rotina dos profissionais de saúde para qualificação da organização da atenção.

\section{REFERÊNCIAS}

1. Ministério da Saúde (BR). Política Nacional de Humanização. Brasília: Ministério da Saúde, 2010.

2. Ministério da Saúde (BR). O trabalho do agente comunitário de saúde. Brasília: Ministério da Saúde, 2009.

3. Ministério da Saúde (BR). Política Nacional de Atenção Básica. Brasília: Ministério da Saúde, 2012.

4. Matumoto S, Mishima SM, Fortuna CM, Pereira MJB, Almeida MCP. Preparando a relação de atendimento: ferramenta para o acolhimento em unidades de saúde. Rev Latino Am Enfermagem. 2009 nov/dez; 17(6):1001-8.

5. Teixeira RR. Acolhimento num serviço de saúde entendido como uma rede de conversações. In: Pinheiro R, Mattos RA. Construção da integralidade: cotidiano, saberes e práticas em saúde. 1 ed. Rio de Janeiro: Cepesc; 2003. p. 91-113.

6. Ministério da Saúde (BR). Política Nacional de Humanização: a humanização como eixo norteador das práticas de atenção e gestão em todas as instâncias do SUS. Brasília: Ministério da Saúde, 2004.

7. Garlet ER, Lima MADS, Santos JLG, Marques GQ. Finalidade do trabalho em urgências e emergências. Rev Latino Am Enfermagem. 2009 jul/ago;17(4):535-40.

8. Santos AE. Humanização em serviços de emergência. In: Calil AM, Paranhos WY, organizadoras. O enfermeiro e as situações de emergência. $2^{a}$. ed. São Paulo: Atheneu; 2010. p. $139-48$.

9. Hiestand B, Moseley M, MacWilliams B, Southwick J. The influence of emergency medical services transport on emergency severity index triage level for patients with abdominal pain. AcadEmerg Med. 2011 mar; 18(3):261-6.

10. Trindade CS. A importância do acolhimento no processo de trabalho das equipes de saúde da família [monografia]. Belo Horizonte: Nescon, Universidade Federal de Minas Gerais; 2010. 39f.

11. Matos LMA. Acolhimento como prática de trabalho para humanizar a assistência no PSF [Internet]. Portal de Educação. 2013 - [acesso em 2016 Jun 19]. Disponível em: http://www.portaleducacao.com.br/enfermagem/artigos/36642/ acolhimento-como-pratica-de-trabalho-para-humanizar-aassistencia-no-psf\#ixzz4ELBXv6yB

12. Zilly A, Calgaro M, Santos MF, Arcoverde MAM, Camargo CRM. Avaliação do acolhimento nas unidades de atenção básica do Paraná. Espaço Saúde - Rev Saúde Púb Paraná 2016; 17(2):206-211. 
13. Rocha NB, Franchin AT, Gasparetto A, Lolli LF, Fujimaki M. Conhecimento sobre acolhimento com classificação de risco pela equipe de atenção básica. Rev Saúde Púb Paraná. 2017 jul; 18(1):72-80.

14. Junior JAB, Matsuda LM. Construção e validação de instrumento para avaliação do Acolhimento com Classificação de Risco. Rev Bras Enferm. 2012 set/out; 65(5):751-7.

15. Versa GLGS, Vituri DW, Buriola AA, Oliveira CA, Matsuda LM. Avaliação do acolhimento com classificação de risco em serviços de emergência hospitalar. Rev Gaúcha Enferm. 2014 set; 35(3):21-28.

16. Lacerda P. O Que é Acolhimento? [Internet]. Brasília. 2016 - [acesso em 2016 Jun 23]. Disponível em: http://www. redehumanizasus.net/94777-o-que-e-acolhimento.

17. Mitre SM, Siqueira BR, Girardi MJM, Morais PNM, Meirelles CAB, Pinto PC, et al. Metodologias ativas de ensinoaprendizagem na formação profissional em saúde: debates atuais. Ciênc Saúde Colet. 2008 dez; 13(2):2133-2144.

18. Prado ML, Velho MB, Espíndola DS, Sobrinho SH, Backes VMS. Arco de Charles Maguerez: refletindo estratégias de metodologia ativa na formação de profissionais de saúde. Esc Anna Nery. 2012 mar; 16 (1):172-177.

19. EPI Info 7 [internet]. Version 7.0. Atlanta: Center for Disease Control and Prevention. [acesso em 2015 Nov 21]. Disponível em: http://www.cdc.gov/epiinfo/.

20. Ayres M, Ayres M Jr, Ayres DL, Santos AS. [internet]. BioEstat: Version 5.0.3. [acesso em 2015 Nov 21]. Disponível em: http://www.mamiraua.org.br/download/index.php?dirpath=./ BioEstat\%205\%20Portugues\&order $=0$.

21. Shiroma LMB, Pires DEP. Classificação de risco em emergência: um desafio para as/os enfermeiras/os. Enferm Foco. 2011 jan;2(1):14-7.

22. Cavalcante RB, Rates HF, Silva LTC, Mello RA, Dayrrel KMB. Acolhimento com classificação de risco: proposta de humanização nos serviços de urgência. Rev Enferm Cent O Min. 2012 set/dez; 2(3):428-37.
23. Acosta AM, Duro CLM, Lima MADS. Atividades do enfermeiro nos sistemas de triagem/classificação de risco nos serviços de urgência: revisão integrativa. Rev Gaúcha Enferm. 2012 dez; 33(4):181-90.

24. Ministério da Saúde (BR). Acolhimento e classificação de risco nos serviços de urgência. Brasília: Ministério da Saúde; 2009.

25. Vituri DW, Matsuda LM. Validação de conteúdo de indicadores de qualidade para avaliação do cuidado de enfermagem. Rev Esc Enferm USP 2009 jun; 43(2):429-37.

26. Silva LG, Alves MS. O acolhimento como ferramenta de práticas inclusivas de saúde. Rev APS. 2008 jan/mar; 11(1):74-84. 